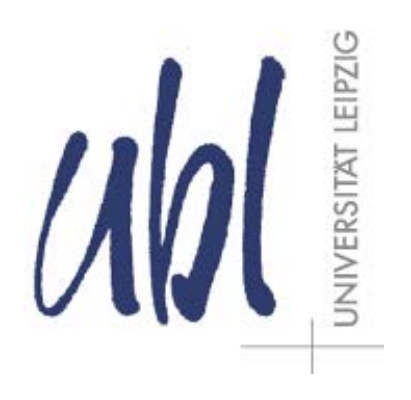

Institute of Medical Physics and Biophysics

Medical Department, Leipzig University

Author Manuscript

(C) 2015. This manuscript version is made available under the CC-BY-NC-ND 4.0 license

http://creativecommons.org/licenses/by-nc-nd/4.0/

Published in final edited form as:

Free Radical Biology and Medicine, 2015 Aug; 85:148-156.

Available at:

http://dx.doi.org/10.1016/j.freeradbiomed.2015.04.015.

\title{
IMPACT OF MYELOPEROXIDASE-DERIVED OXIDANTS ON THE PRODUCT PROFILE OF HUMAN 5-LIPOXYGENASE
}

\author{
Josefin Zschaler ${ }^{a, b}$, Juliane Dorow ${ }^{c, d}$, Louisa Schöpe ${ }^{a}$, Uta Ceglarek ${ }^{c, d}$, Jürgen \\ Arnhold ${ }^{a, b}$ \\ a Institute for Medical Physics and Biophysics, Medical Faculty, Leipzig \\ University, Leipzig, Germany \\ b Translational Centre for Regenerative Medicine Leipzig, Leipzig University, \\ Leipzig, Germany \\ c Institute of Laboratory Medicine, Clinical Chemistry and Molecular \\ Diagnostics, University Hospital Leipzig, Leipzig, Germany \\ d LIFE - Leipzig Research Center for Civilization Diseases, Universität Leipzig, \\ Leipzig, Germany
}

\section{Highlights}

- Myeloperoxidase-derived oxidants changed the product profile of 5-lipoxygenase.

- The ratio of 5-HpETE to 6-trans- $\mathrm{LTB}_{4}$ increased with increasing $\mathrm{HOCl}$ and $\mathrm{HOBr}$.

- Epoxidation of 5-HpETE was more strongly affected than conversion of arachidonic acid.

- 5-Lipoxygenase also produced 8- and 12-HpETE after incubation with $\mathrm{HOCl}$ and $\mathrm{HOBr}$.

- The whole myeloperoxidase system showed effects similar to those of $\mathrm{HOCl}$ and $\mathrm{HOBr}$.

\section{Abstract}

Human 5-lipoxygenase (5-LOX) oxidizes arachidonic acid to 5S-hydroperoxy-6E,8Z,11Z,14Zeicosatetraenoic acid (5-HpETE) and leukotriene (LT) $A_{4}$. In neutrophils, LTA $_{4}$ is further converted to the potent chemoattractant $\mathrm{LTB}_{4}$. These cells also contain the heme enzyme myeloperoxidase (MPO), which produces several potent oxidants such as hypochlorous acid $(\mathrm{HOCl})$, which are involved in pathogen defense and immune regulation. Here, we 
addressed the question whether MPO-derived oxidants are able to affect the activity of 5-LOX and the product profile of this enzyme. Human 5-LOX was incubated with increasing amounts of $\mathrm{HOCl}$ or $\mathrm{HOBr}$. Afterward, arachidonic acid metabolites of 5-LOX were analyzed by reverse-phase high-performance liquid chromatography as well as by liquid chromatography-electrospray ionization-tandem mass spectrometry. The incubation of 5-LOX with the MPO-derived oxidants significantly changed the product profile of 5-LOX. Thereby, $\mathrm{HOCl}$ and $\mathrm{HOBr}$ increased the ratio of $5-\mathrm{H}(\mathrm{p}) \mathrm{ETE}$ to 6-trans- $\mathrm{LTB}_{4}$ in a concentration-dependent manner. At low oxidant concentrations, there was a strong decrease in the yield of 6-trans- $\mathrm{LTB}_{4}$, whereas 5-HpETE did not change or increased. Additionally, the formation of 8-HpETE and 12 -HpETE by 5-LOX rose slightly with increasing $\mathrm{HOCl}$ and $\mathrm{HOBr}$. Comparable results were obtained with the $\mathrm{MPO}-\mathrm{H}_{2} \mathrm{O}_{2}-\mathrm{Cl}^{-}$system when glucose oxidase and glucose were applied as a source of $\mathrm{H}_{2} \mathrm{O}_{2}$. This was necessary because of a strong impairment of 5-LOX activity by $\mathrm{H}_{2} \mathrm{O}_{2}$. In summary, MPO-derived oxidants showed a considerable impact on 5-LOX, impairing the epoxidation of 5-HpETE, whereas the hydroperoxidation of arachidonic acid was unaffected. Apparently, this was caused by an oxidative modification of critical amino acid residues of 5-LOX. Further work is necessary to assess the specific type and position of oxidation in the substrate-binding cavity of 5-LOX and to specify whether this interaction between 5-LOX and MPO-derived oxidants also takes place in stimulated neutrophils.

\section{Keywords}

Eicosanoids, Arachidonic acid, $\mathrm{HOCl}, \mathrm{HOBr}$, Neutrophils, Free radicals

\section{Introduction}

During the innate immune response, polymorphonuclear leukocytes, also called neutrophils, are the first effector cells that will be recruited from the peripheral blood to inflammatory loci. These cells are equipped with an arsenal of protecting systems able to recognize, inactivate, and destroy foreign microorganisms. Enzymes and signaling systems will be sequentially activated in neutrophils during their interaction with the inflamed endothelium, during diapedesis and migration of these cells through the endothelial layer and adjacent space, as well as finally during recognition and phagocytosis of microorganisms [Kolaczkowska and Kubes 2013].

In this respect, 5-lipoxygenase (5-LOX), a non-heme-iron dioxygenase that uses arachidonic acid (AA) as substrate, is crucial for the chemotactic movement of neutrophils. This enzyme is also present in eosinophils, monocytes/macrophages, dendritic cells, and mast cells [Radmark et al. 2015]. Equimolar amounts of hydroperoxides can activate 5-LOX by oxidation of the inactive ferrous iron to the ferric form [Rouzer and Samuelsson 1986]. Afterward, the lipoxygenase reaction is started by stereoselective hydrogen abstraction at C7 of AA. Then, the resulting radical migrates to the C5 position, where it reacts with dioxygen forming a peroxyl radical. In a subsequent interaction with the ferrous form of 5-LOX, a peroxyl anion is generated yielding 5S-hydroperoxy-6E,8Z,11Z,14Zeicosatetraenoic acid (5-HpETE) after protonation [Haeggstrom and Funk 2011]. Human 5-LOX also possesses a secondary leukotriene $A_{4}\left(\right.$ LTA $\left._{4}\right)$ synthase activity [Rouzer et al. 1986]. Thereby, a hydrogen at C10 of 5-HpETE is abstracted followed by a radical migration to the $\mathrm{C} 6$ position, rearrangement of double bonds, and formation of an epoxide moiety [Maas et al. 1982]. In neutrophils the unstable $\mathrm{LTA}_{4}$ is thereafter metabolized to leukotriene $\mathrm{B}_{4}\left(\mathrm{LTB}_{4}\right)$ by the action of LTA $_{4}$ hydrolase [Newcomer and Gilbert 2010]. 
Leukotriene $\mathrm{B}_{4}$, a potent autocrine chemoattractant to these cells, is released at the moving site of neutrophils where it acts on specific receptors and causes an actin contraction in the adjacent cell compartment [McDonald et al. 1994].

The heme protein myeloperoxidase (MPO) is highly specific to neutrophils. It has also been found to a lesser extent in monocytes. Myeloperoxidase is present in the azurophilic granules that will fuse with phagocytosed bacteria. Constituents of azurophilic granules contribute to the inactivation and killing of microorganisms [Klebanoff et al. 2013]. Myeloperoxidase is known to be activated by hydrogen peroxide producing an arsenal of active oxidants including hypochlorous acid $(\mathrm{HOCl})$ and hypobromous acid $(\mathrm{HOBr}$ ) [Arnhold and Flemmig 2010]. Under physiological conditions especially $\mathrm{HOCl}$ is generated in neutrophils. Typical reaction partners of $\mathrm{HOCl}$ are thiols, thioethers, and amino groups [Hawkins et al. 2003]. Therefore, $\mathrm{HOCl}$ is principally able to oxidize amino acid residues of proteins, such as cysteine, methionine, tyrosine, tryptophan, and lysine residues [Pattison and Davies 2001]. Furthermore, various studies showed chemical modifications of certain proteins [Bouriche et al. 2007; Malle et al. 2006; Salavej et al. 2006].

Both enzymes, 5-LOX and MPO, get sequentially activated during migration of neutrophils to inflammatory loci. An increased $\mathrm{Ca}^{2+}$ level during neutrophil activation leads to translocation of cytosolic 5-LOX to the nuclear membrane and in consequence to the synthesis of $\mathrm{LTB}_{4}$, promoting chemotaxis of these cells [Radmark et al. 2015]. At the final destination of neutrophils, $\mathrm{HOCl}$ production via MPO is activated through stronger stimulation. Here, the question arises whether $\mathrm{HOCl}$, a potent amino acid-oxidizing agent, could affect the activity or the product profile of 5-LOX. Previously, effects of a possible interaction of $\mathrm{HOCl}$ with $\mathrm{AA}$-derived hydroperoxides were investigated [Zschaler and Arnhold 2014]. Here, the hydroperoxide moiety was not affected by $\mathrm{HOCl}$, and chlorohydrins were formed instead. However, in the presence of a potential reaction partner of $\mathrm{HOCl}$, such as the thioether group of methionine, the formation of chlorohydrins was disturbed [Zschaler and Arnhold 2014]. Thus, 5-LOX could be a better target site of $\mathrm{HOCl}$.

The aim of this study was to assess the effects of $\mathrm{HOCl}$ on the activity and product profile of 5-LOX. Analysis was performed by reverse-phase high-performance liquid chromatography (RP-HPLC) as well as by liquid chromatography-electrospray ionization-tandem mass spectrometry (LC-ESI-MS/MS). Furthermore, hydrogen peroxide, also generated during neutrophil activation; hypobromous acid, the equivalent of $\mathrm{HOCl}$ in eosinophil granulocytes; and finally activated MPO were evaluated according to their impact on 5-LOX. We could demonstrate that $\mathrm{HOCl}$, either present as a reagent or formed by the myeloperoxidasehydrogen peroxide-chloride system, and $\mathrm{HOBr}$ considerably affect the product profile of 5-LOX, without, however, disturbing the lipoxygenase activity. 


\section{Material and methods}

\section{Material}

Human 5-LOX was purchased from MyBioSource (supplier A, Catalog No. MBS537729, San Diego, CA, USA) or Cayman Chemical distributed by Biomol (supplier B, Catalog No. Cay60402, Hamburg, Germany). In both cases the recombinant enzyme was isolated from a baculovirus overexpression system in Sf21 cells and the S100 supernatant was used after centrifugation $(100,000 \mathrm{~g})$. A dialysis was performed against phosphate buffer $(50 \mathrm{mM}, \mathrm{pH}$ 7.4) to replace the Tris buffer of the commercial 5-LOX solution using a Slide-A-Lyzer dialysis cassette (3500 Da molecular mass cut-off, Thermo Scientific).

The chemicals and further enzymes used were obtained from the following sources: human neutrophil myeloperoxidase from Planta (Vienna, Austria); glucose oxidase from bovine erythrocytes (G6137) from Sigma (Taufkirchen, Germany); HPLC standards 5-HpETE, 5Shydroxy-6E,8Z,11Z,14Z-eicosatetraenoic acid (5-HETE), 5S,12R-dihydroxy-6E,8E,10E,14Zeicosatetraenoic acid (5,12-DiHETE), 12S-hydroperoxy-5Z,8Z,10E,14Z-eicosatetraenoic acid (12-HpETE), 15S-hydroperoxy-5Z,8Z,11Z,13E-eicosatetraenoic acid (15-HpETE), 13Shydroperoxy-9Z,11E-octadecadienoic acid (13-HpODE), and AA were from Cayman Chemical (distributed by Biomol); HPLC solvents were from Carl Roth (Karlsruhe, Germany); and all other chemicals were from Sigma.

Working solutions of $\mathrm{HOCl}$ and $\mathrm{H}_{2} \mathrm{O}_{2}$ were prepared by dilution of the corresponding stock solutions. Their concentrations were determined spectrophotometrically using $\varepsilon_{292}=350 \mathrm{M}^{-1} \mathrm{~cm}^{-1}$ at $\mathrm{pH} 12$ in $1 \mathrm{~N}$ sodium hydroxide solution for ${ }^{-} \mathrm{OCl}$ [Morris 1966] and $\varepsilon_{240}=43.6 \mathrm{M}^{-1} \mathrm{~cm}^{-1}$ for $\mathrm{H}_{2} \mathrm{O}_{2}$ [Beers and Sizer 1952]. The synthesis of $\mathrm{HOBr}$ was performed by the reaction of $\mathrm{HOCl}$ with bromide, whereby $40 \mathrm{mM} \mathrm{NaBr}$ was mixed with $20 \mathrm{mM} \mathrm{HOCl}$ in phosphate buffer $\left(50 \mathrm{mM}, \mathrm{pH}\right.$ 7.4). The concentration of ${ }^{-} \mathrm{OBr}$ was measured spectrophotometrically using $\varepsilon_{329}=332 \mathrm{M}^{-1} \mathrm{~cm}^{-1}$ at $\mathrm{pH} 12$ in $1 \mathrm{~N}$ sodium hydroxide solution [Kumar and Margerum 1987]. The solutions were essentially stable for $1 \mathrm{~h}$ at $4{ }^{\circ} \mathrm{C}$ and were used within this time.

\section{Incubation of human 5-LOX with various oxidants}

Dialyzed recombinant human 5-LOX (6 U) was incubated with various oxidants $(\mathrm{HOCl}, \mathrm{HOBr}$, or $\mathrm{H} 2 \mathrm{O} 2)$ at $20^{\circ} \mathrm{C}$ in phosphate buffer $(50 \mathrm{mM}, \mathrm{pH} 7.4)$ for $5 \mathrm{~min}(\mathrm{~V}=80 \mu \mathrm{l})$ under shaking conditions (40 rpm). Afterward, 5-LOX activity assay was performed by addition of final $100 \mu \mathrm{M}$ AA, $5 \mu \mathrm{M}$ 13-HpODE, $100 \mu \mathrm{M}$ EDTA, $300 \mu \mathrm{M}$ Ca2+ and $200 \mu \mathrm{M}$ ATP and incubation for further $10 \mathrm{~min}\left(V_{\text {final }}=100 \mu \mathrm{l}\right)$. The reaction was terminated by the addition of $200 \mu \mathrm{l}$ ice-cold acetonitrile and $3 \mu \mathrm{l}$ acetic acid (100\%) and the precipitate was separated by centrifugation $\left(10 \mathrm{~min}, 4^{\circ} \mathrm{C}, 10,000 \mathrm{~g}\right)$. The supernatants were stored at $-80^{\circ} \mathrm{C}$ and analyzed by RP-HPLC within $24 \mathrm{~h}$.

\section{Incubation of human 5- $\mathrm{LOX}$ with the $\mathrm{MPO}-\mathrm{H}_{2} \mathrm{O}_{2}-\mathrm{Cl}^{-}$system}

Dialyzed recombinant human 5-LOX (6 U) was incubated with $200 \mathrm{nM} \mathrm{MPO,} 140 \mathrm{mM} \mathrm{NaCl}$, $400 \mathrm{mU}$ glucose oxidase, and $30-140 \mu \mathrm{M}$ glucose at $20^{\circ} \mathrm{C}$ in phosphate buffer $(50 \mathrm{mM}, \mathrm{pH}$ 7.4) for $15 \mathrm{~min}(\mathrm{~V}=80 \mu \mathrm{l})$ under shaking conditions ( $40 \mathrm{rpm})$. Glucose oxidase and glucose were added to ensure a steady supply of low amounts of $\mathrm{H}_{2} \mathrm{O}_{2}$ and consequently activate MPO to produce $\mathrm{HOCl}$. The synthesized $\mathrm{HOCl}$ was measured using a 3,3',5,5'tetramethylbenzidine (TMB)-based assay [Dypbukt et al. 2005]. Afterward, the 5-LOX 
activity assay was performed (see Incubation of human 5-LOX with various oxidants). Finally, samples were stored at $-80^{\circ} \mathrm{C}$ and analyzed by RP-HPLC within $24 \mathrm{~h}$.

\section{Analysis of 5-LOX products by RP-HPLC}

The samples were analyzed by RP-HPLC using a $\mathrm{C}_{18}$ column (Supelcosil LC-18-DB, $25 \mathrm{~cm} \times$ $4.6 \mathrm{~mm}$ i.d., $5 \mu \mathrm{m}$ ), with an isocratic eluent consisting of acetonitrile/ $\mathrm{H}_{2} \mathrm{O} /$ acetic acid $(60 / 40 / 0.2, v / v / v)$ with a flow rate of $1 \mathrm{ml} / \mathrm{min}$. The eluate was monitored at $234 \mathrm{~nm}$ (quantification of conjugated dienes) and $270 \mathrm{~nm}$ (quantification of conjugated trienes). The HPLC consisted of a Shimadzu liquid chromatographic system equipped with a Shimadzu LC-10ATvp isocratic solvent delivery system, Shimadzu SPD-10Avp dualwavelength absorbance detector, Shimadzu CTO-10ASvp column oven $\left(35^{\circ} \mathrm{C}\right)$, and Rheodyne injector with $20 \mu \mathrm{l}$ loop volume. The concentrations of the LOX products (5-H(p)ETE, 6-trans-LTB 4 , 12-HpETE) were quantified using a calibration curve (13-point calibration) of the appropriate synthetic standards. In the case of 8-HpETE no synthetic standard was available, therefore the 12-HpETE calibration curve was used instead. The identification of 8-HpETE was performed by MS/MS analysis after RP-HPLC separation. Therefore, a mixture of hydroperoxides of AA was synthesized using controlled $\alpha$-tocopherol-inhibited autoxidation of AA [Peers and Coxon 1983]. Afterward, this mixture was separated by RP-HPLC as described above. Thereby 5-HETE showed a shoulder at a lower retention time. This peak was collected and analyzed by mass spectrometry. According to its fragmentation pattern this peak could be attributed to 8-HpETE [MacMillan and Murphy 1995].

\section{Analysis of HOCl-modified 5-LOX products by LC-ESI-MS/MS}

Human 5-LOX (6 U) was incubated with $\mathrm{HOCl}$ as described under Incubation of human 5LOX with various oxidants. The reaction was terminated by dilution (1:500 and 1:5000) in phosphate buffer $(50 \mathrm{mM}, \mathrm{pH} 7.4)$ and addition of $50 \mu \mathrm{l}$ internal standard solution and $400 \mu \mathrm{l}$ precipitation reagent containing methanol:zinc sulfate $4: 1(\mathrm{v} / \mathrm{v})$ to $200 \mu \mathrm{l}$ of the corresponding sample. After $2 \mathrm{~min}$ vortexing the samples were centrifuged for $5 \mathrm{~min}$ at $10,000 \mathrm{~g}$ and transferred in HPLC vials for storage at $-80^{\circ} \mathrm{C}$. LC-ESI-MS/MS was performed according to Kortz et al. [Kortz et al. 2013]. In brief, $200 \mu \mathrm{l}$ of the sample solution was injected onto a Strata-X extraction column $(20 \times 2 \mathrm{~mm}$ i.d., $25 \mu \mathrm{m}$, Phenomenex, Aschaffenburg, Germany) for online solid-phase extraction and chromatographically separated on a Kinetex C18 column ( $100 \times 2.1 \mathrm{~mm}$ i.d., $2.6 \mu \mathrm{m}$, Phenomenex). A 5500 QTrap mass spectrometer (AB Sciex, Darmstadt, Germany) in negative ionization mode was applied for $\mathrm{MS} / \mathrm{MS}$ analysis with scheduled multiple-reaction monitoring (MRM) experiments. For detection of the 5-LOX metabolite 6-trans-LTB 4 at the retention time of 4.3 $\mathrm{min}$ the MRM $\mathrm{m} / \mathrm{z} 335.2 / 194.9$ was included in the experiment. Quantification of 6-trans-LTB ${ }_{4}$ was performed using the $\mathrm{LTB}_{4}$ calibration curve (internal standard: $\mathrm{LTB}_{4}-\mathrm{d}_{4}$ ). 


\section{Results}

\section{$\mathrm{HOCl}$ affects the product profile of human 5-LOX}

Arachidonic acid was converted by human 5-LOX into two major products, 5-HpETE and $\mathrm{LTA}_{4}$. 5-HpETE resulted from the dioxygen insertion at the C5 position of AA and can be reduced spontaneously or in vitro by a peroxidase-catalyzed reduction into 5-HETE due to the instability of the hydroperoxide group. Leukotriene $A_{4}$ was rapidly nonenzymatically hydrolyzed into 6-trans-leukotriene $\mathrm{B}_{4}$ (6-trans-LTB $\mathrm{L}_{4}$ ). Accordingly, chromatographic analysis of AA oxidation by 5-LOX revealed 5-HpETE $(c=28.7 \mu \mathrm{M}), 5$-HETE $(c=6.1 \mu \mathrm{M})$, and 6-trans- $\mathrm{LTB}_{4}(\mathrm{C}=20.1 \mu \mathrm{M})$ (Figure $1 \mathrm{~A}$ and $\mathrm{B}$, black line) as main products.

Afterward, the influence of $\mathrm{HOCl}$ on the enzyme activity and product profile of 5-LOX was assessed. Thus, 5-LOX was incubated with increasing amounts of $\mathrm{HOCl}$ before the substrate mixture was added (Figure 1).
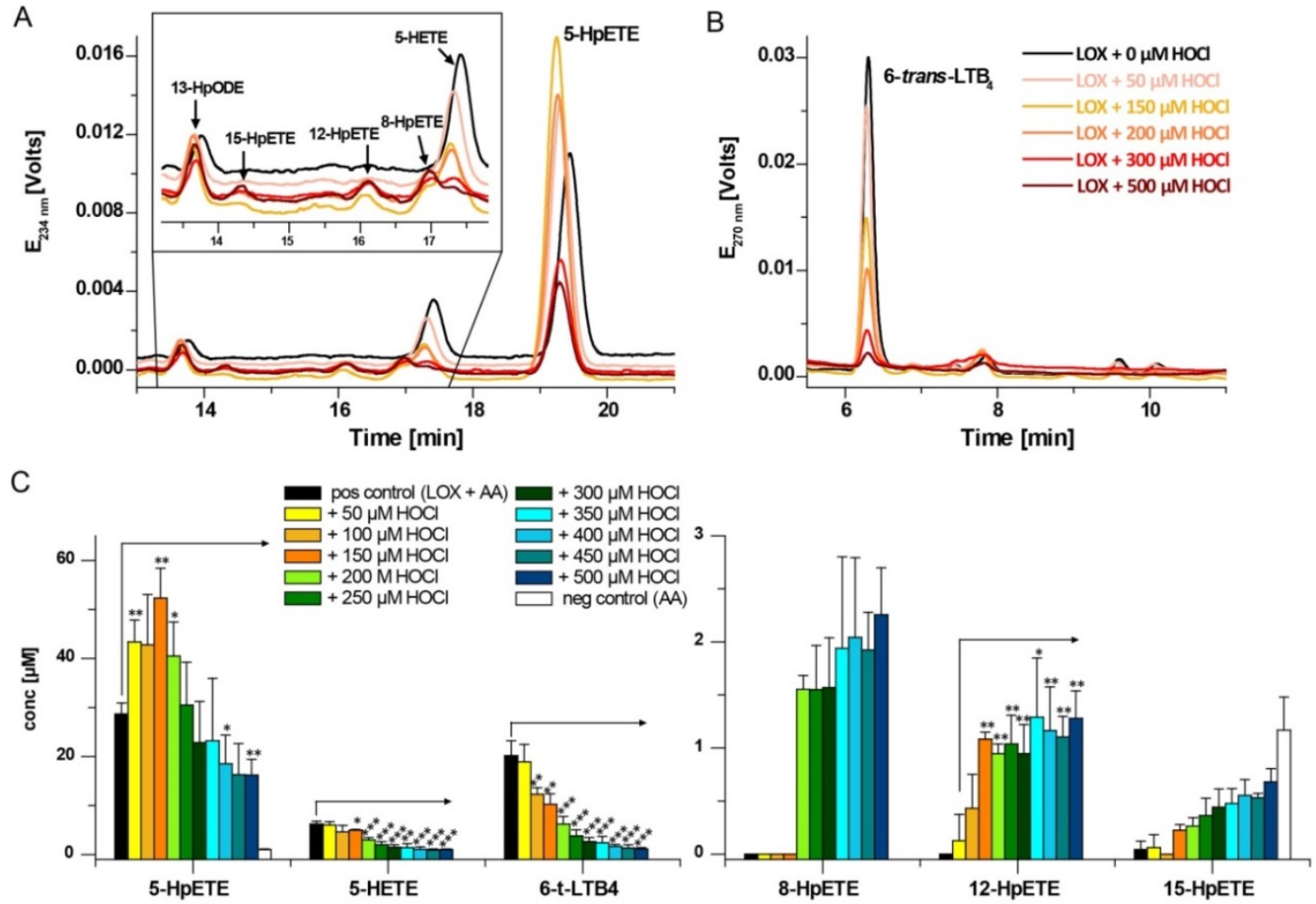

Figure 1: Recombinant human 5-LOX (6 U, $46.5 \mathrm{U} / \mathrm{mg}$, supplier A) was incubated with $\mathrm{HOCl}$ at $20{ }^{\circ} \mathrm{C}$ in phosphate buffer (50 mM, pH 7.4) for 5 min. Afterward, $100 \mu \mathrm{M} \mathrm{AA}, 5 \mu \mathrm{M}$ 13-HpODE, $100 \mu \mathrm{M}$ EDTA, $300 \mu \mathrm{M} \mathrm{Ca}^{2+}$, and $200 \mu \mathrm{M}$ ATP were added and incubated for a further $10 \mathrm{~min}$. The reaction was terminated by protein precipitation and the samples were analyzed by RP-HPLC at (A) $234 \mathrm{~nm}$ and (B) $270 \mathrm{~nm}$. The concentrations of (C) 5-HpETE, 5-HETE, and 6-trans-LTB 4 and 8-HpETE, 12-HpETE, and 15-HpETE were calculated using a calibration curve of synthetic standards. pos/neg, positive/negative; two-tailed $t$ test against positive control, ${ }^{*} p \leq 0.05,{ }^{*} p \leq 0.01,{ }^{*} * \mathrm{p} \leq 0.001 ; \mathrm{n}=3$ or 4 .

A significant increase in the 5-HpETE peak was observed at $\mathrm{HOCl}$ concentrations up to $200 \mu \mathrm{M}$ (Figure $1 \mathrm{~A}$ and C). Only at $\mathrm{HOCl}$ concentrations of $400 \mu \mathrm{M}$ or higher, the yield of 5-HpETE was lower than in the absence of $\mathrm{HOCl}$. Interestingly, the peaks of 6-trans- $\mathrm{LTB}_{4}$ and 5-HETE decreased over the whole $\mathrm{HOCl}$ concentration range (Figure $1 \mathrm{~A}, \mathrm{~B}$, and $\mathrm{C}$ ). Surprisingly, two further AA-derived products were detected after incubation of 5-LOX with $\mathrm{HOCl}$, resulting from the oxygenation at $\mathrm{C} 12$ and $\mathrm{C} 8$ of $\mathrm{AA}$ (Figure $1 \mathrm{~A}$, inset; Figure $1 \mathrm{C}$ ). 
However, the maximal concentrations of $12-\mathrm{HpETE}\left(\mathrm{c}_{\max }=1.3 \mu \mathrm{M}\right)$ and $8-\mathrm{HpETE}\left(\mathrm{c}_{\max }=2.3\right.$ $\mu \mathrm{M})$ were rather low.

The AA solution was also measured as a negative control without the addition of 5-LOX (Figure $1 \mathrm{C}$, white bar). Here, low intense peaks for 15-HpETE and 5-HpETE were detected owing to nonspecific oxidation of the AA stock solution. Further nonspecific oxidation products such as $12 / 8-\mathrm{HpETE}$ were not detected. A peroxide-free AA could not be used because of antioxidative additives interfering with $\mathrm{HOCl}$. However, in the untreated 5-LOX samples $15-\mathrm{HpETE}$ was not detected as a product (Figure $1 \mathrm{C}$, black bar). Possibly, low amounts of AA hydroperoxides were consumed during 5-LOX activation in addition to the applied 13-HpODE. After $\mathrm{HOCl}$ addition, low intense peaks were detected representing $15-\mathrm{HpETE}\left(\mathrm{c}_{\max }=0.7 \mu \mathrm{M}\right)$. It could not be evaluated whether this $15-\mathrm{HpETE}$ was produced by 5 -LOX or derived from the AA stock solution.

The $\mathrm{HOCl}$-induced changes in 5-LOX products revealed drastic differences between 5-HpETE on one hand and 5-HETE and 6-trans-LTB 4 on the other (Figure $2 \mathrm{~A}$ ). After application of $150 \mu \mathrm{M} \mathrm{HOCl}$ a 1.76-fold increase in the 5-HpETE concentration took place, whereas a 1.27and 1.86-fold decrease occurred for 5-HETE and 6-trans-LTB 4 , respectively. Apparently, low amounts of $\mathrm{HOCl}$ already blocked further conversions of 5-HpETE and caused, thus, a rise in 5-HpETE production.
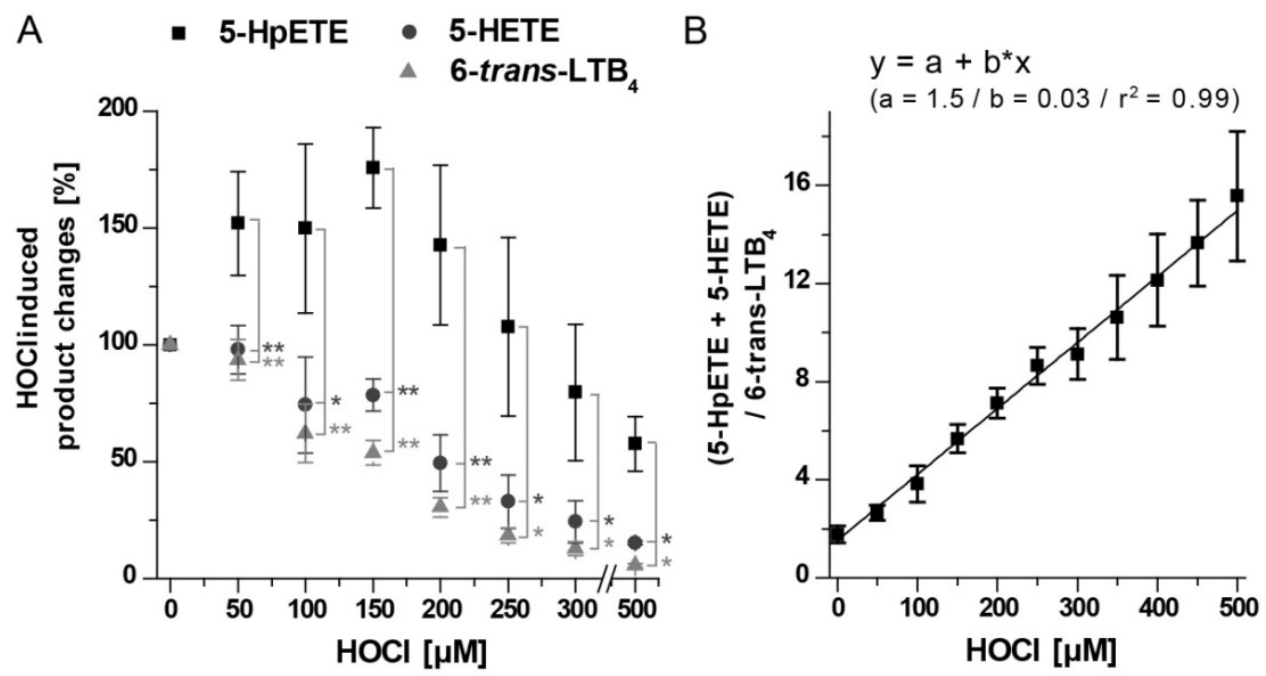

Figure 2: $\mathrm{HOCl}$-induced alterations in the product profile of human 5-LOX as described for Figure 1. (A) The $\mathrm{HOCl}$-induced changes in the main products are related in percentage to the applied $\mathrm{HOCl}$ concentrations. Two-tailed $t$ test against 5-HpETE, ${ }^{*} p \leq 0.05,{ }^{*} p \leq 0.01$. (B) The ratio of 5-H(p)ETE to 6-trans-LTB 4 was related to the applied $\mathrm{HOCl}$ concentration. $\mathrm{n}=3$ or 4 .

Taking into account that 5-H(p)ETE derived from the first oxygenation of AA by 5-LOX, and 6-trans- $\mathrm{LTB}_{4}$ from the subsequent oxygenation, the ratio of $5-\mathrm{H}(\mathrm{p}) \mathrm{ETE}$ to 6-trans-LTB indicates the prevalence of the respective step (Figure $2 \mathrm{~B}$ ). There was a linear increase in the 5-H(p)ETE to 6-trans-LTB 4 ratio from 1.8:1 to 15.6:1 when 5-LOX was incubated with increasing $\mathrm{HOCl}$ concentrations. Hence, especially $5-\mathrm{HpETE}$ was the dominating product of HOCl-modified 5-LOX, whereas the further conversion of 5-HpETE to $\mathrm{LTA}_{4}$ was strongly impaired. 


\section{Products of HOCl-modified 5-LOX analyzed by LC-ESI-MS/MS}

The product profile of 5-LOX after treatment with $\mathrm{HOCl}$ was further analyzed by LC-ESIMS/MS. This method enables a fast quantification of a broad spectrum of oxidized metabolites. In addition to the main products 5-H(p)ETE and 6-trans-LTB ${ }_{4}$, other further AAderived oxygenation products were detected, namely 5,6-DiHETE, 8-HETE, 12-HpETE, and 5-OXo-ETE (Figure $3 \mathrm{~A}$ ). However, their concentrations were rather low. 12-HpETE and 8-HpETE were also measured using RP-HPLC (see $\mathrm{HOCl}$ affects the product profile of human 5-LOX). For 8-HpETE no commercial standard was available, therefore it could not be measured with the applied LC-ESI-MS/MS. Nevertheless, the reduction product 8-HETE indicates the presence of 8-HpETE. Both 5,6-DiHETE and 5-oxo-ETE are further nonenzymatic hydrolysis products of $\mathrm{LTA}_{4}$ even though they emerged in lower amounts than the 5,12-DiHETE form (6-trans-LTB ${ }_{4}$ ) [Borgeat and Samuelsson 1979; Gravel et al. 1993]. Both products were also detected using RP-HPLC; however, their concentrations were negligible compared to 6-trans-LTB 4 .

The most significant $\mathrm{HOCl}$-induced changes in the 5-LOX products arose for 6-trans- $\mathrm{LTB}_{4}$, 5-HETE, and 5,6-DiHETE (Figure $3 \mathrm{~A}$ ), where a strong decrease occurred. Also a decline was obvious for 5-oxo-ETE, albeit not to the same extent. These alterations are comparable to the previous experiment, also with regard to the further LTA $_{4}$ hydrolysis products, 5,6-DiHETE and 5-oxo-ETE.
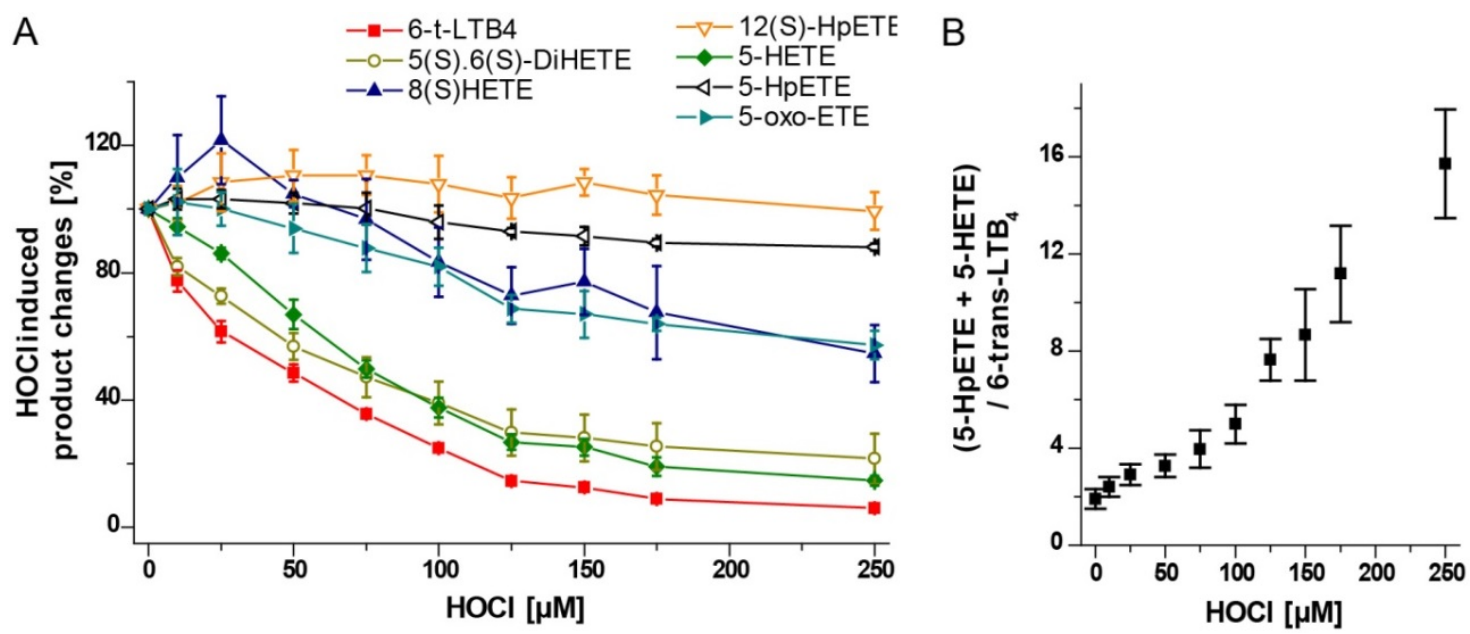

Figure 3: The incubation of 5-LOX $(6 \mathrm{U}, 162.9 \mathrm{U} / \mathrm{mg}$, supplier A) with $\mathrm{HOCl}$ and the following activity assay were performed as described for Figure 1 . The samples were analyzed by LC-ESI-MS/MS. (A) Concentrations of the main products were calculated using a calibration curve of synthetic standards. (B) The ratio of 5-H(p)ETE to 6-trans-LTB 4 was related to the applied $\mathrm{HOCl}$ concentration. $\mathrm{n}=3$ or 4 .

The HOCl-induced effects on 5-HpETE correspond only partly to the results of the RP-HPLC analysis. The amount of 5-HpETE remained relatively stable after treatment of 5-LOX with $\mathrm{HOCl}$. A comparable increase in the concentration at low $\mathrm{HOCl}$ did not occur. Also 12-HpETE showed only a modest $\mathrm{HOCl}$-induced increase. However, here $12-\mathrm{HpETE}$ belonged also to the untreated 5-LOX product profile. In the previous results 5-LOX produced 12-HpETE only after $\mathrm{HOCl}$ incubation (see $\mathrm{HOCl}$ affects the product profile of human 5-LOX). This slightly changed product profile can be explained with another enzyme charge used. Interestingly, for 8-HETE an initial rise in the concentration was detected, decreasing with higher $\mathrm{HOCl}$ amounts. 
It has to be mentioned that there was a strong quantitative difference. Using the RP-HPLC a six times higher concentration of 5-HpETE was measured compared to the LC-ESI-MS/MS procedure. Also for 5-HETE and 6-trans-LTB 4 lower amounts were detected with LC-ESIMS/MS. Apparently, the different precipitation procedure, necessary for LC-MS/MS measurement, affected the yield of 5-HpETE and diminished the effects of the $\mathrm{HOCl}$ induced increase. Nevertheless, when 5-LOX was incubated with $\mathrm{HOCl}$ in a concentration range from 10 to $250 \mu \mathrm{M} \mathrm{HOCl}$ the ratio of 5-H(p)ETE to 6-trans-LTB 4 showed a comparable strong increase, from 1.9:1 to 15.7:1 (Figure $3 \mathrm{~B}$ ). In the previous experiments, $\mathrm{HOCl}$ was tested in a concentration range between 50 and $500 \mu \mathrm{M}$ leading to an increase in the ratio of 5-H(p)ETE to 6-trans-LTB ${ }_{4}$ from 1.8:1 to 15.6:1 (Figure 1). These differences in $\mathrm{HOCl}$ concentration can be attributed to the different enzyme charges used, affecting the required $\mathrm{HOCl}$ amount for a comparable effect.

\section{Inactivation of human 5-LOX by $\mathrm{H}_{2} \mathrm{O}_{2}$}

The product profile of $\mathrm{HOCl}$-treated 5-LOX was extensively changed, especially concerning the ratio of 5-HpETE to $\mathrm{LTA}_{4}$. However, it is not clear if a slight inactivation of the enzyme happens after $\mathrm{HOCl}$ incubation. Therefore, the effect of $\mathrm{HOCl}$ on the product profile of 5-LOX was compared to $\mathrm{H}_{2} \mathrm{O}_{2}$ as a further reactive oxygen species. For this purpose, 5-LOX was incubated with $\mathrm{H}_{2} \mathrm{O}_{2}$ under conditions comparable to those of $\mathrm{HOCl}$. Afterward, the substrate mixture was added and the product profile of 5-LOX was assessed by RP-HPLC (Figure 4).
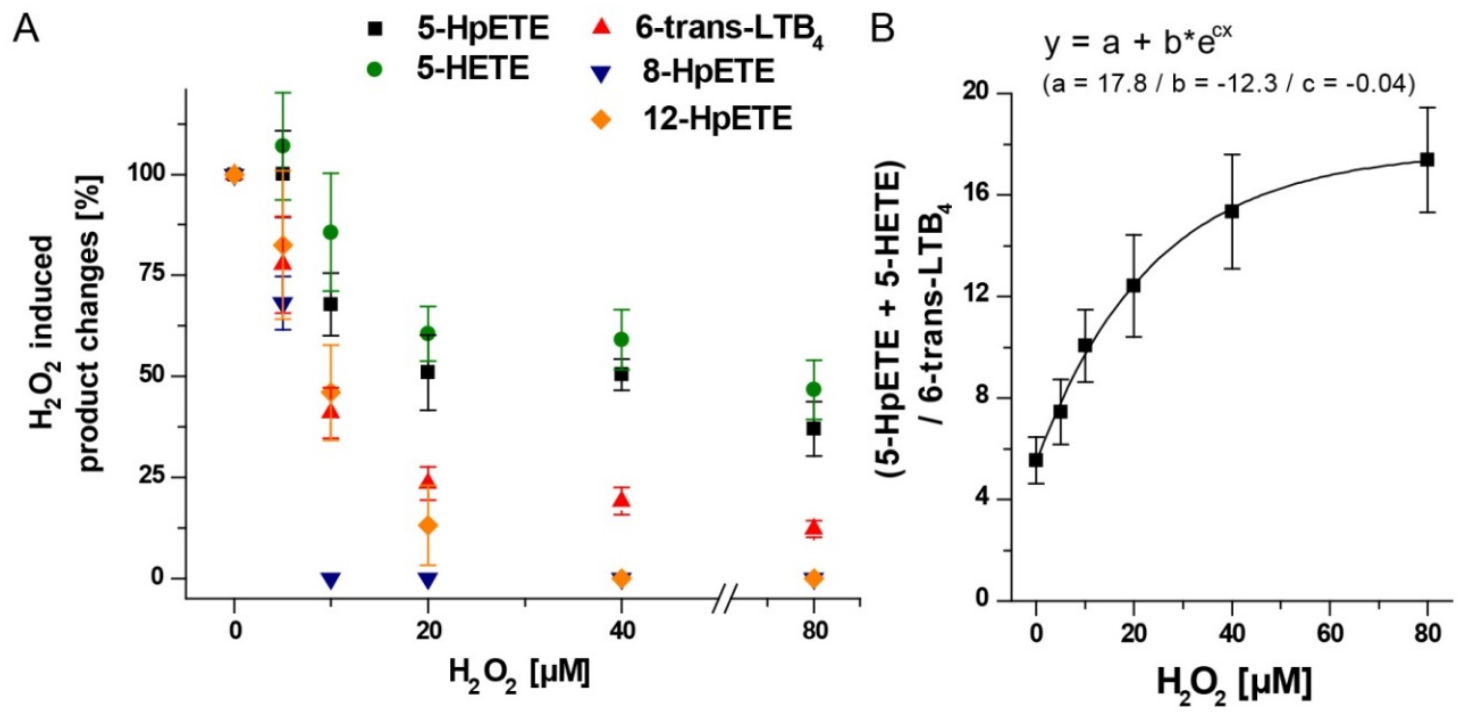

Figure 4: Recombinant 5-LOX $\left(6 \mathrm{U}, 34.4 \mathrm{U} / \mathrm{mg}\right.$, supplier B) was incubated with $\mathrm{H}_{2} \mathrm{O}_{2}$ at $20{ }^{\circ} \mathrm{C}$ in phosphate buffer (50 mM, pH 7.4) for $5 \mathrm{~min}$. Afterward, an activity assay of 5-LOX and chromatographic quantification of the product profile was performed and $(A) \mathrm{H}_{2} \mathrm{O}_{2}$-induced changes in percentage of the product profile of human 5-LOX were calculated. (B) The ratio of 5-H(p)ETE to 6-trans- $\mathrm{LTB}_{4}$ was related to the applied $\mathrm{H}_{2} \mathrm{O}_{2}$ concentration. $\mathrm{n}=3$ or 4 .

With increasing amounts of $\mathrm{H}_{2} \mathrm{O}_{2}$ both 5-HpETE and 5-HETE, as well as 6-trans- $\mathrm{LTB}_{4}$, displayed a substantial decrease especially in the range from $5 \mu \mathrm{M}$ to $20 \mu \mathrm{M} \mathrm{H} \mathrm{H}_{2} \mathrm{O}_{2}$ (Figure $4 \mathrm{~A})$. For 6 -trans- $\mathrm{LTB}_{4}$ a considerable decline of $77 \%$ was observed but also concentrations of 5-HETE and 5-HpETE were markedly reduced by 39 and $49 \%$, respectively. Higher $\mathrm{H}_{2} \mathrm{O}_{2}$ concentrations up to $80 \mu \mathrm{M}$ resulted in only slightly further changes. Interestingly, 12-HpETE and 8-HpETE, detected as clear signals with the enzyme preparation used at 3.2 and $2.9 \mu \mathrm{M}$, respectively, completely diminished up to $20 \mu \mathrm{M} \mathrm{H} \mathrm{H}_{2}$. This highlights 
clearly the different actions of $\mathrm{HOCl}$ and $\mathrm{H}_{2} \mathrm{O}_{2}$ in relation to 5-LOX, where both these oxidation products increased in their concentration after $\mathrm{HOCl}$ treatment. The $\mathrm{H}_{2} \mathrm{O}_{2}-$ induced changes in the ratio of $5-\mathrm{H}(\mathrm{p}) \mathrm{ETE}$ to 6 -trans- $-\mathrm{LTB}_{4}$ were also determined (Figure $4 \mathrm{~B})$. It became evident that also for $\mathrm{H}_{2} \mathrm{O}_{2}$-treated 5-LOX this ratio increased from 5.5:1 to 17.4:1. This could be explained by the faster decrease in 6-trans-LTB 4 compared to 5-H(p)ETE. However, for $\mathrm{HOCl}$ a linear correlation between $\mathrm{HOCl}$ and 5- $\mathrm{H}(\mathrm{p}) \mathrm{ETE}$ to 6-trans$\mathrm{LTB}_{4}$ occurred, whereas $\mathrm{H}_{2} \mathrm{O}_{2}$ induced an exponential rise. This was apparently caused by the stronger effect in the range between 5 and $20 \mu \mathrm{M} \mathrm{H} \mathrm{H}_{2} \mathrm{O}_{2}$.

\section{HOBr affects the product profile of human 5-LOX}

A further prominent hypohalous acid produced from peroxidases is hypobromous acid. $\mathrm{HOBr}$ was also assessed according to its effects on the product profile of 5-LOX. Here, $\mathrm{HOBr}$ was incubated in the range from 10 to $250 \mu \mathrm{M}$ with 5-LOX before the enzyme activity was determined (Figure 5).
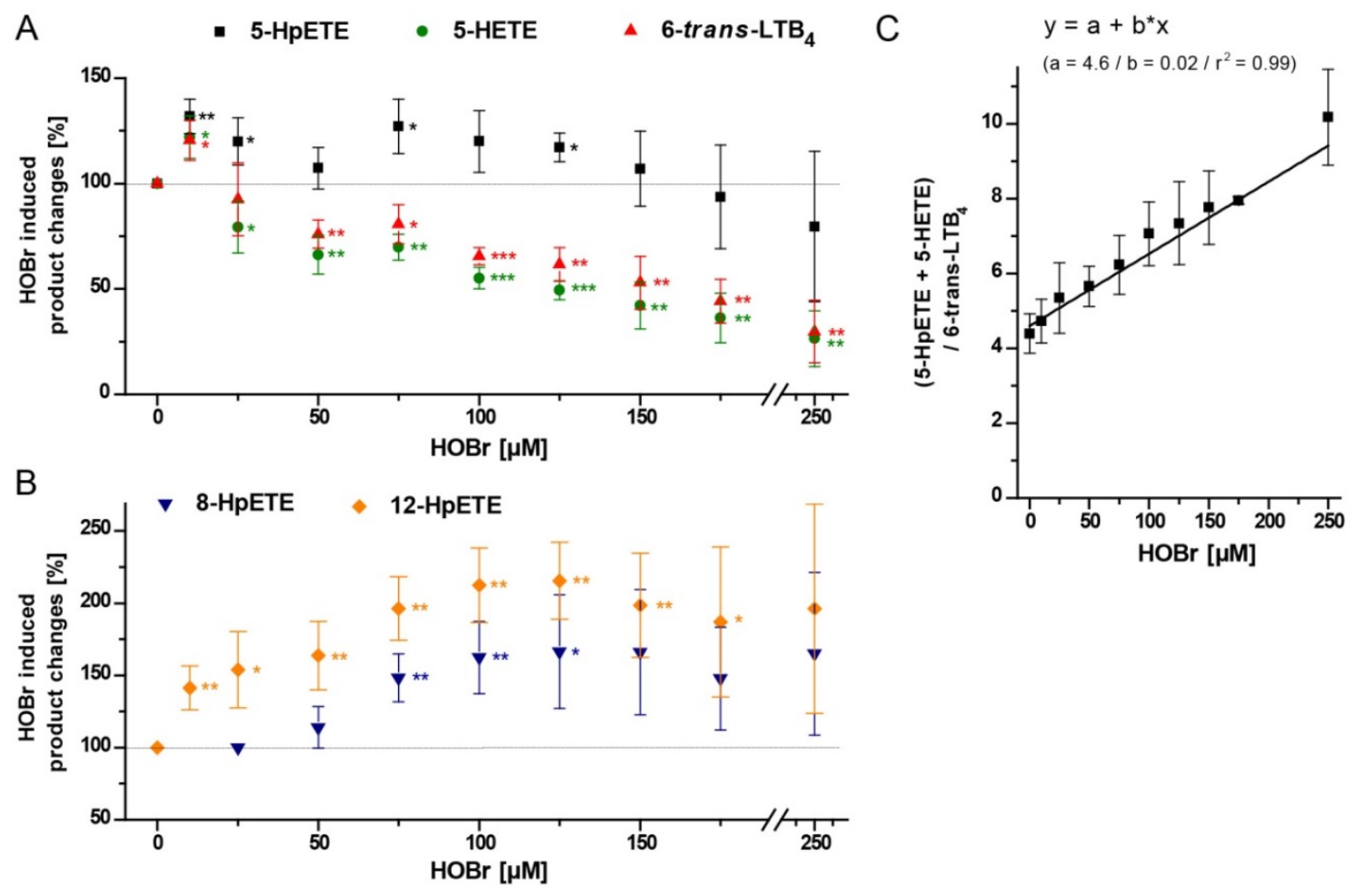

Figure 5: Recombinant 5-LOX (6 U, $34.4 \mathrm{U} / \mathrm{mg}$, supplier B) was incubated with $\mathrm{HOBr}$ at $20{ }^{\circ} \mathrm{C}$ in phosphate buffer (50 mM, pH 7.4) for 5 min. Afterward, an activity assay of 5-LOX and chromatographic quantification of the product profile were performed and $\mathrm{HOBr}$-induced changes in percentage of the product profile of human 5-LOX were calculated for (A) 5-H(p)ETE and 6-trans-LTB 4 and (B) 8-HpETE and 12-HpETE. (C) The ratio of 5-H(p)ETE to 6-trans- $\mathrm{LTB}_{4}$ was related to the applied $\mathrm{HOBr}$ concentration. Two-tailed $t$ test against control, ${ }^{*} \mathrm{p} \leq 0.05,{ }^{* *} \mathrm{p} \leq 0.01,{ }^{* * *} \mathrm{p} \leq 0.001 ; \mathrm{n}=4$.

The lowest $\mathrm{HOBr}$ concentration induced an increase in all metabolites. However, higher amounts of $\mathrm{HOBr}$ led to a significant reduction in 6-trans-LTB 4 and 5-HETE, whereas for 5-HpETE a significantly higher concentration was still present (Figure 5 A). For example, 125 $\mu \mathrm{M} \mathrm{HOBr}$ induced a 1.17-fold increase in 5-HpETE and a decrease of 49 and $62 \%$ for 5 -HETE and 6-trans-LTB $\mathrm{LT}_{4}$, respectively. Interestingly, a distinctive $\mathrm{HOBr}$-induced augmentation was observed for 8-HpETE and 12-HpETE. Here, $125 \mu \mathrm{M} \mathrm{HOBr}$ caused a 1.67- and 2.16-fold increase in $8-\mathrm{HpETE}$ and $12-\mathrm{HpETE}$, respectively (Figure $5 \mathrm{~B}$ ). Furthermore, the ratio of 
5-H(p)ETE to 6-trans-LTB 4 was determined (Figure $5 \mathrm{C}$ ) showing a linear dependence on the applied $\mathrm{HOBr}$ concentration. Unmodified 5-LOX had a ratio of 5-H(p)ETE to 6-trans-LTB ${ }_{4}$ of 4.4:1, growing up to $10.1: 1$ after addition of $250 \mu \mathrm{M} \mathrm{HOBr}$. This was comparable to the $\mathrm{HOCl}$-induced 5-LOX effects. However, for unmodified 5-LOX (see $\mathrm{HOCl}$ affects the product profile of human 5-LOX) a lower ratio of 5-H(p)ETE to 6-trans-LTB 4 was determined, which can be attributed to the different enzyme charges used.

\section{Interaction of activated MPO and human 5-LOX}

The heme-containing protein $\mathrm{MPO}$ produces $\mathrm{HOCl}$ in neutrophil granulocytes after activation by hydrogen peroxide. The MPO- $\mathrm{H}_{2} \mathrm{O}_{2}-\mathrm{Cl}^{-}$system was now applied to modify the product profile of human 5-LOX. As demonstrated above $\mathrm{H}_{2} \mathrm{O}_{2}$ easily inactivates 5-LOX (see Inactivation of human 5-LOX by $\mathrm{H}_{2} \mathrm{O}_{2}$ ). Therefore, a simple addition of $\mathrm{H}_{2} \mathrm{O}_{2}$ was not possible for MPO activation in the presence of 5-LOX. The glucose oxidase/glucose (GOD/Glu) system was used instead, to ensure a steady supply of low amounts of $\mathrm{H}_{2} \mathrm{O}_{2}$. Different glucose concentrations were added to glucose oxidase to achieve different degrees of MPO activation and subsequently variable $\mathrm{HOCl}$ concentrations. The efficiency of the MPO$\mathrm{GOD} / \mathrm{Glu}^{-\mathrm{Cl}^{-}}$system was tested by $\mathrm{HOCl}$ quantification using a TMB-based assay. Thereby, half of the applied glucose was converted into $\mathrm{HOCl}$ (data not shown). Afterward, 5-LOX was incubated with MPO and the GOD/Glu system (Figure 6). As controls $\mathrm{Cl}^{-}$was omitted as MPO substrate or the GOD/Glu system alone was added to 5-LOX. Here, a strong decrease in the concentrations of all metabolites was observed. For example, addition of $100 \mu \mathrm{M}$ glucose to the 5-LOX/MPO/GOD samples devoid of $\mathrm{Cl}^{-}$resulted in a 3.3- and 10.9-fold decrease for 5-HpETE and 6-trans- $\mathrm{LTB}_{4}$, respectively. This was comparable to the $\mathrm{H}_{2} \mathrm{O}_{2}$ effect seen earlier (Inactivation of human 5-LOX by $\mathrm{H}_{2} \mathrm{O}_{2}$ ). Also 12-HpETE and 8-HpETE produced from unaffected 5-LOX completely disappeared in these controls. The addition of $\mathrm{Cl}^{-}$to 5-LOX, MPO, and the GOD/Glu system also resulted in a reduction in 5-HETE and 6-trans-LTB 4 . Also the amount of 5-HpETE was attenuated; however, only to a minor degree compared to 6-trans-LTB 4 . Here, $100 \mu \mathrm{M}$ glucose led to a 1.2- and 3.1-fold decrease for 5-HpETE and 6-trans-LTB $\mathrm{LB}_{4}$, respectively. The small changes in the 5-HpETE concentration might be due to the $\mathrm{H}_{2} \mathrm{O}_{2}$-driven impairment of 5-LOX activity. This could superimpose the HOCl-induced 5-HpETE increase. Interestingly, the amount of 12-HpETE and 8-HpETE was unaffected in the 5-LOX, MPO, $\mathrm{Cl}^{-}$, and GOD/Glu samples, clearly distinguishing them from the negative controls in which 5-LOX was influenced by the $\mathrm{H}_{2} \mathrm{O}_{2}$-generating system. 


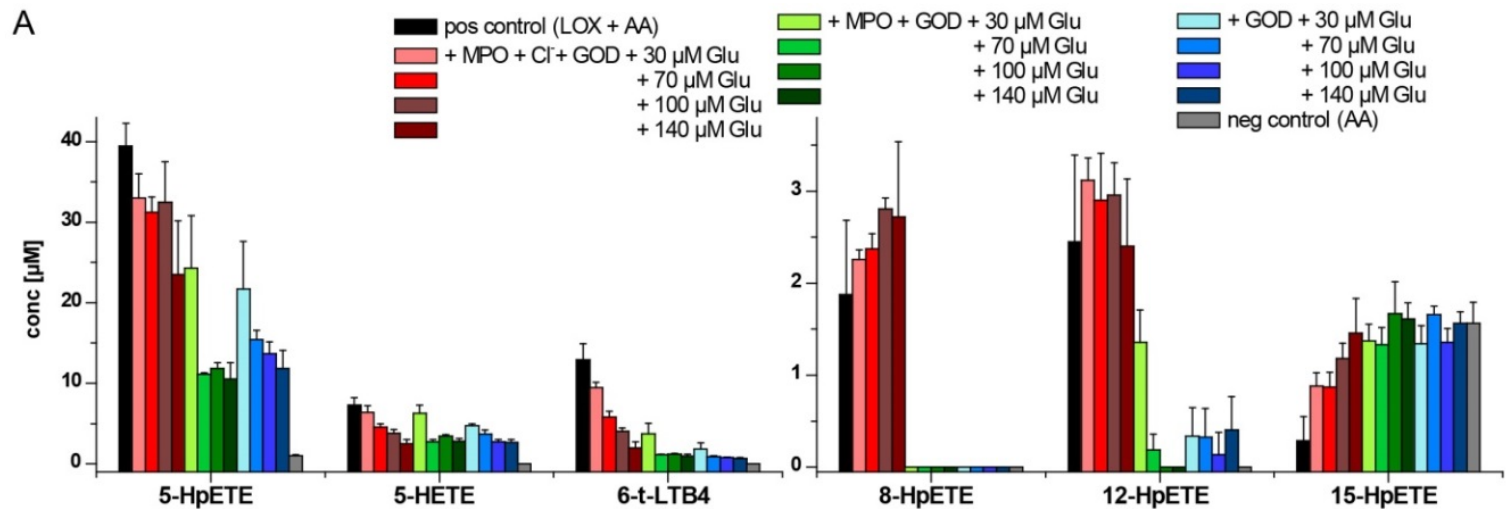

B

- 5-HpETE • 5-HETE $\Delta$ 6-trans-LTB 4

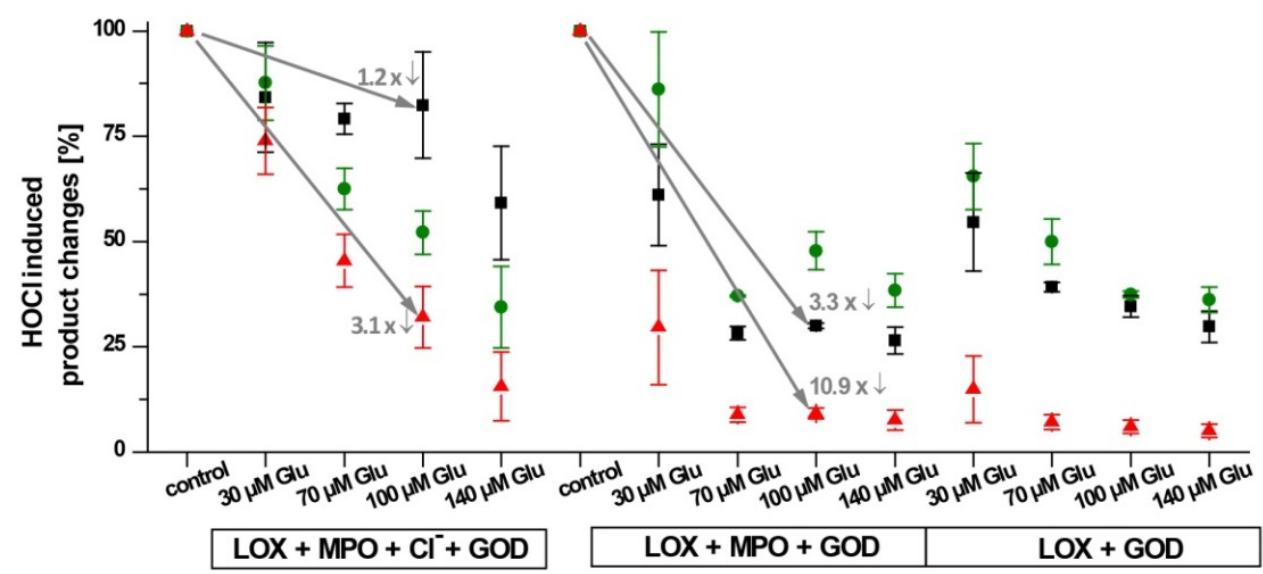

Figure 6: Recombinant human 5-LOX (6 U, $162.9 \mathrm{U} / \mathrm{mg}$, supplier A) was incubated with $200 \mathrm{nM}$ MPO, $400 \mathrm{mU}$ glucose oxidase, and various amounts of glucose at $20^{\circ} \mathrm{C}$ in phosphate buffer $(50 \mathrm{mM}$, $\mathrm{pH}$ 7.4) for $15 \mathrm{~min}(\mathrm{~V}=80 \mu \mathrm{l})$. Afterward an activity assay of 5-LOX and chromatographic quantification of the product profile were performed. The concentrations of (A) 5-HpETE, 5-HETE, and 6-trans-LTB 4 and (B) 8-HpETE, 12-HpETE, and 15-HpETE were calculated using a calibration curve of synthetic standards. (C) $\mathrm{HOCl}$-induced changes in percentage of the product profile of human 5LOX after incubation with activated MPO (MPO $\left.+\mathrm{Cl}^{-}+\mathrm{GOD}\right)$ and further negative controls with MPO $+\mathrm{GOD}$ in the absence of $\mathrm{Cl}^{-}$and sole addition of the GOD/Glu system to 5-LOX. pos/neg, positive/negative; GOD, glucose oxidase; Glu, glucose, $n=3$.

As shown in the previous experiments the ratio of 5-H(p)ETE to 6-trans-LTB 4 was determined (Figure 7). An exponential increase in this ratio occurred for both 5-LOX/MPO/GOD samples devoid of $\mathrm{Cl}^{-}$(from 3.7:1 to 14.0:1) and 5-LOX/GOD samples (from 3.7:1 to 22.7:1) depending on the added glucose concentration, which is comparable to the inactivation of 5-LOX with $\mathrm{H}_{2} \mathrm{O}_{2}$ (see Inactivation of human 5-LOX by $\mathrm{H}_{2} \mathrm{O}_{2}$ ). The addition of $\mathrm{Cl}^{-}$to 5-LOX, MPO, and the GOD/Glu system led in turn to a slower rise in the ratio of 5-H(p)ETE to 6-trans-LTB 4 (from 3.7:1 to 14.0:1). 


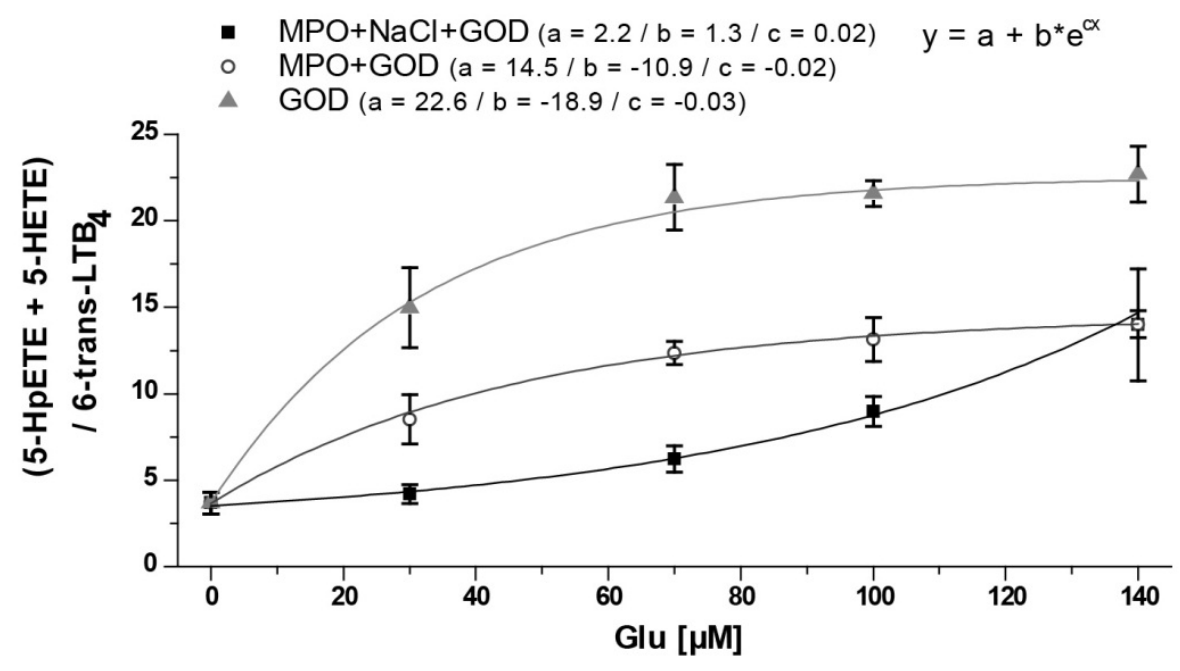

Figure 7: Recombinant human 5-LOX (6 U) was incubated with MPO, glucose oxidase, and various amounts of glucose (see Figure 6). Afterward, an activity assay of 5-LOX and chromatographic quantification of the product profile were performed. The ratio of 5-H(p)ETE to 6-trans-LTB4 was related to the applied glucose concentration. GOD, glucose oxidase; Glu, glucose. 


\section{Discussion}

5-Lipoxgenase is known to catalyze two successive reactions in the formation of potent bioactive metabolites derived from AA. First, this enzyme oxidizes AA into 5-HpETE and converts in a second step this hydroperoxide into LTA 4 . Whereas the formation of 5-HpETE and 5-HETE, the reduced form of 5-HpETE, could be directly verified with our methods, the formation of the unstable $\mathrm{LTA}_{4}$ was evaluated assessing its nonenzymatic hydrolysis product 6-trans- $\mathrm{LTB}_{4}$.

The incubation of 5-LOX with the MPO-derived oxidants $\mathrm{HOCl}$ and $\mathrm{HOBr}$ significantly changed the product profile of 5-LOX in a concentration-dependent manner. There was a linear increase in the ratio of $5-\mathrm{H}(\mathrm{p}) \mathrm{ETE}$ to 6 -trans- $\mathrm{LTB}_{4}$ with increasing concentrations of $\mathrm{HOCl}$ and $\mathrm{HOBr}$. At low oxidant concentrations, there was a strong decrease in the yield of 6-trans- $\mathrm{LTB}_{4}$, whereas 5-HpETE did not change or increased. 5-HpETE was decreased only at higher concentrations for $\mathrm{HOCl}$ and $\mathrm{HOBr}$. Incubation of 5- $\mathrm{LOX}$ with $\mathrm{H}_{2} \mathrm{O}_{2}$ diminished all

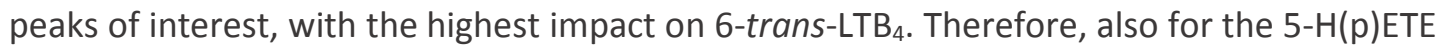
to 6-trans- $\mathrm{LTB}_{4}$ ratio an increase was obvious with increasing $\mathrm{H}_{2} \mathrm{O}_{2}$ concentration, but not in a linear manner. Thus, whereas small MPO-related oxidants mostly disturb the conversion of 5-HpETE into LTA $\mathrm{LA}_{4}$ b-LOX, $\mathrm{H}_{2} \mathrm{O}_{2}$ impaired the whole 5-LOX activity. A reaction of $\mathrm{HOCl}$ and $\mathrm{HOBr}$ with the AA-derived products itself can be ruled out. Previous studies showed that the double bonds are a target site for $\mathrm{HOCl}$, but only in the absence of further reaction partners such as the thioether group of methionine [Zschaler and Arnhold 2014].

We applied two 5-LOX preparations from different suppliers. These preparations differed in their $5-\mathrm{H}(\mathrm{p}) \mathrm{ETE}$ to 6 -trans- $\mathrm{LTB}_{4}$ ratio of the unperturbed sample. The initial ratios were 1.8 to 3.7 and 4.4 to 5.5 for supplier $A$ and $B$, respectively (see Results). We can only speculate about the reasons for these differences. It is clear that the addition of $\mathrm{HOCl}$ or $\mathrm{H}_{2} \mathrm{O}_{2}$ to 5-LOX leads to an increased 5-H(p)ETE to 6-trans-LTB 4 ratio. Whether the increased ratio of the untreated 5-LOX is related to a preoxidized enzyme preparation could not been examined.

The 5-H(p)ETE to 6-trans- $\mathrm{LTB}_{4}$ ratio increased also after incubation of 5-LOX with the MPO$\mathrm{H}_{2} \mathrm{O}_{2}-\mathrm{Cl}^{-}$system. Owing to the high impact of $\mathrm{H}_{2} \mathrm{O}_{2}$ on 5-LOX, glucose oxidase/glucose was applied as a source of $\mathrm{H}_{2} \mathrm{O}_{2}$. This reduced considerably the actual concentrations of hydrogen peroxide over a bolus addition. According to the results for $\mathrm{H}_{2} \mathrm{O}_{2}$, the product ratio of $5-\mathrm{H}(\mathrm{p}) \mathrm{ETE}$ and 6 -trans-LTB $\mathrm{LT}_{4}$ increased also in a nonlinear manner using glucose oxidase/glucose alone or with MPO in the absence of chloride. However, the complete MPO system had a much lower impact on the product profile of 5-LOX.

A second observation was the increased formation of 8-HpETE and 12-HpETE in 5-LOX samples incubated with $\mathrm{HOCl}, \mathrm{HOBr}$, and the $\mathrm{MPO}-\mathrm{H}_{2} \mathrm{O}_{2}-\mathrm{Cl}^{-}$system, which could not be detected after incubation of 5-LOX with $\mathrm{H}_{2} \mathrm{O}_{2}$. Instead, $\mathrm{H}_{2} \mathrm{O}_{2}$ or the GOD/Glu system disturbed the primarily produced 8-HpETE and 12-HpETE formation. In addition to 5-HpETE these hydroperoxides are well known to be produced by 5-LOX with a lower yield [Walther et al. 2009], which could be verified by our results. We further observed an increased formation of these products with increasing $\mathrm{HOCl}$ and $\mathrm{HOBr}$ concentrations.

In two different studies human 5-LOX was converted into a 15-lipoxygenating enzyme by mutational introduction of a space-filling amino acid, reducing the volume of the substrate cavity, or by mimicking the Ser663 phosphorylation site [Gilbert et al. 2012; Schwarz et al. 
2001]. We measured a slightly increasing peak for 15-HpETE in HOCl-influenced 5-LOX samples compared to the untreated sample. Even though 15-HpETE was already obvious in the AA stock solution, apparently owing to autoxidation, in untreated 5-LOX samples no 15-HpETE was present. Probably, 15-HpETE reacts during the enzymatic activity assay with ferrous 5-LOX during lipoxygenase activation, comparable to 13-HpODE [Riendeau et al. 1989; Schilstra et al. 1992]. Now, the slightly increased HOCl-dependent 15-HpETE peak could be due to an effect of $\mathrm{HOCl}$ on the general 5-LOX activity, leading to lower enzyme activation and thus a reduced consumption of $15-\mathrm{HPETE}$ introduced via the AA stock solution. However, low concentrations of $\mathrm{HOCl}$ did not disturb the 5-HpETE-producing activity of 5-LOX, assuming that 5-LOX activation is comparable. Therefore, it is more possible that in addition to the changed oxidation specificity, leading to $12-\mathrm{HpETE}$ and 8-HpETE, 15-HpETE is also produced by 5-LOX.

The influence of $\mathrm{HOCl}$ on the product profile of 5-LOX was also analyzed with LC-ESIMS/MS, a sophisticated technique to measure a high number of oxidized metabolites in a relatively short time. In principal, a comparable $\mathrm{HOCl}$ effect was detected, leading to a linear increase in the 5-H(p)ETE to 6-trans-LTB 4 ratio. However, the quantitative results could not be compared. Here, for 5-HpETE and 6-trans-LTB 4 , a 6.1- and 3.8-fold lower concentration was measured with LC-ESI-MS/MS compared to RP-HPLC, respectively, whereas for 5-HETE only a 1.2-fold lower concentration was obvious. This could be reasoned by the application of different precipitation procedures, the lack of the appropriate internal standard for 5-HpETE quantification, or the instability of 5-HpETE during electrospray ionization diminishing the effects of the $\mathrm{HOCl}$-induced increase in 5-HpETE.

In addition to 6-trans-LTB LTd $_{4}$ and 6-DiHETE as nonenzymatic hydrolysis products of $\mathrm{LTA}_{4}$ [Gravel et al. 1993], 5-oxo-ETE was also measured in the LOX samples using the LC-ESIMS/MS technique. 5-Oxo-ETE could be nonspecifically formed from the decomposition of 5-HpETE during the preparation or after injection onto the column, resulting in 5-oxo$6 E, 8 Z, 11 Z, 14 Z$-eicosatetraenoic acid. This phenomenon was reported for the injection of 12-HpETE or 15-HpETE onto an HPLC column without prior reduction [Fruteau de Laclos and Borgeat 1988]. However, the measured product might also be 5-oxo-7E,9E,11Z,14Zeicosatetraenoic acid, a further nonenzymatic hydrolysis product of $\mathrm{LTA}_{4}$ [Gravel et al. 1993], differing only in the position of the double bonds. Gravel et al. described an MS/MS fragment with $\mathrm{m} / \mathrm{z} 203$ Da for 5-oxo-7E,9E,11Z,14Z-eicosatetraenoic acid [Gravel et al. 1993], which is also used for the applied LC-ESI-MS/MS analysis of 5-oxo-ETE. Thus, the triene structure (5-oxo-7E,9E,11Z,14Z-eicosatetraenoic acid) is thought to have the same retention time as the stereoisomer 5-oxo-6E,8Z,11Z,14Z-eicosatetraenoic acid in the LC-ESI$\mathrm{MS} / \mathrm{MS}$ analysis.

5-LOX possesses two functions during leukotriene biosynthesis, hydroperoxidation and epoxidation, certainly differing in their catalytic mechanism [Rouzer et al. 1986]. The hydroperoxidation is initiated by pro-S hydrogen abstraction at C7 of $A A$, whereas epoxidation proceeds after abstraction of the pro-R hydrogen at $\mathrm{C} 10$, suggesting a different position of the substrate relative to the ferric active center [Haeggstrom and Funk 2011]. Furthermore, the resulting radical reacts only in the hydroperoxidation step with molecular dioxygen to a peroxyl radical species, which is subsequently reduced by ferrous iron. In contrast, during epoxidation the hydroperoxide group is homolytically cleaved by ferrous iron, yielding a hydroxide radical for epoxidation. Recently detailed kinetic investigations of 
these two processes were performed, indicating that both the rate of substrate capture and the rate of product release are less efficient for epoxidation than for hydroperoxidation [Smyrniotis et al. 2014]. Furthermore, it was assumed that for 5-LOX it is more efficient to retain the 5-HpETE in its active site for sequential epoxidation, rather than binding free 5-HpETE [Smyrniotis et al. 2014]. Therefore, the impact of the MPO-derived oxidants on the product profile of 5-LOX could be related to the oxidation of critical amino acid residues in the substrate binding cavity disturbing mostly the less efficient epoxidation of 5-HpETE, whereas the hydroperoxidation of AA is unaffected. Further, 5-HpETE is more compact and more hydrophilic than AA. Thus, slight changes in the conformation of the substrate cavity due to amino acid oxidation could result in an impaired radical formation at C10 or the disturbance of a further step during epoxidation.

In addition, the crystal structure of human 5-LOX revealed a unique Phe, Tyr-cork feature blocking the possible entrance to the active site [Gilbert et al. 2011]. Modification of these two amino acids could result in a changed entrance mechanism, affecting the uptake of AA and 5-HpETE in a different way. However, at the moment, we can only speculate as to which fine mechanisms take place. To verify these differences, it is highly necessary to combine our measurements with proteomic analysis for any oxidant-mediated modifications in the protein structure of 5-LOX.

Hydrogen peroxide affected both reactions catalyzed by 5-LOX, hydroperoxidation and epoxidation, with a stronger impact on the formation of LTA $_{4}$. As explained above, this might be related to the less efficient epoxidation reaction. However, $\mathrm{H}_{2} \mathrm{O}_{2}$ clearly diminished the whole 5-LOX activity, assuming a direct impact on the active center of the 5-LOX. The inactivation mechanism could involve a reaction between $\mathrm{H}_{2} \mathrm{O}_{2}$ and ferrous iron [Percival et al. 1992]. Furthermore, the iron ligand histidines are potential target sites for metal-catalyzed oxidation reactions [Dean et al. 1989].

Changes in the product profile of 5-LOX were investigated in in vitro enzyme preparations. It is highly necessary to extend these measurements to neutrophil granulocytes containing both 5-LOX and MPO. Alternatively, eosinophils are also an interesting target as they contain two lipoxygenases, namely 5-LOX and 15-LOX, and eosinophil peroxidase producing $\mathrm{HOBr}$. The activation of 5-LOX in granulocytes is an early event in inflammation and immune response. In neutrophils, $\mathrm{LTB}_{4}$ is an important chemotactic agent, necessary for the directed movement of these cells to inflammatory loci. Enhanced oxidant formation by MPO is mostly related to phagocytosis of microorganisms and their digestion in phagosomes. Apparently, MPO-derived oxidants play a certain role in terminating signals for the formation of $\mathrm{LTB}_{4}$.

\section{Declaration of Interest}

The authors have no conflicts of interest to declare. The authors alone are responsible for the content and writing of the paper.

\section{Acknowledgement}

The authors gratefully acknowledge the financial support provided by the Sächsische Aufbaubank through funding from the European Regional Development Fund (SAB Project No. 100116526) and the German Federal Ministry of Education and Research (BMBF 1315883). 


\section{References}

Arnhold J, Flemmig J (2010) Human myeloperoxidase in innate and acquired immunity. Arch Biochem Biophys 500, 92-106.

Beers RF, Sizer I (1952) A spectrophotometric method for measuring the breakdown of hydrogen peroxide by catalase. J Biol Chem 195, 133-40.

Borgeat P, Samuelsson B (1979) Metabolism of arachidonic acid in polymorphonuclear leukocytes. Structural analysis of novel hydroxylated compounds. J Biol Chem 254, 7865-9.

Bouriche H, Salavei P, Lessig J, Arnhold J (2007) Differential effects of flavonols on inactivation of alpha1-antitrypsin induced by hypohalous acids and the myeloperoxidase-hydrogen peroxide-halide system. Arch Biochem Biophys 459, 137-42.

Dean RT, Wolff SP, McElligott MA (1989) Histidine and proline are important sites of free radical damage to proteins. Free Radic Res Commun 7, 97-103.

Dypbukt JM, Bishop C, Brooks WM, Thong B, Eriksson H, Kettle AJ (2005) A sensitive and selective assay for chloramine production by myeloperoxidase. Free Radic Biol Med 39, 1468-77.

Fruteau de Laclos B, Borgeat P (1988) Conditions for the formation of the oxo derivatives of arachidonic acid from platelet 12-lipoxygenase and soybean 15-lipoxygenase. Biochim Biophys Acta 958, 424-33.

Gilbert NC, Bartlett SG, Waight MT, Neau DB, Boeglin WE, Brash AR, Newcomer ME (2011) The structure of human 5-lipoxygenase. Science 331, 217-9.

Gilbert NC, Rui Z, Neau DB, Waight MT, Bartlett SG, Boeglin WE, Brash AR, Newcomer ME (2012) Conversion of human 5-lipoxygenase to a 15-lipoxygenase by a point mutation to mimic phosphorylation at Serine-663. FASEB J 26, 3222-9.

Gravel J, Falgueyret JP, Yergey J, Trimble L, Riendeau D (1993) Identification of 5-keto(7E,9E,11Z,14Z)-eicosatetraenoic acid as a novel nonenzymatic rearrangement product of leukotriene A4. Arch Biochem Biophys 306, 469-75.

Haeggstrom JZ, Funk CD (2011) Lipoxygenase and leukotriene pathways: biochemistry, biology, and roles in disease. Chem Rev 111, 5866-98.

Hawkins CL, Pattison DI, Davies MJ (2003) Hypochlorite-induced oxidation of amino acids, peptides and proteins. Amino Acids 25, 259-74.

Klebanoff SJ, Kettle AJ, Rosen H, Winterbourn CC, Nauseef WM (2013) Myeloperoxidase: a front-line defender against phagocytosed microorganisms. J Leukoc Biol 93, 185-98.

Kolaczkowska E, Kubes P (2013) Neutrophil recruitment and function in health and inflammation. Nat Rev Immunol 13, 159-75.

Kortz L, Dorow J, Becker S, Thiery J, Ceglarek U (2013) Fast liquid chromatography-quadrupole linear ion trap-mass spectrometry analysis of polyunsaturated fatty acids and eicosanoids in human plasma. J Chromatogr B Analyt Technol Biomed Life Sci 927, 209-13.

Kumar K, Margerum DW (1987) Kinetics and mechanism of general-acid-assisted oxidation of bromide by hypochlorite and hypochlorous acid. Inorg Chem 26, 2706-11.

Maas RL, Ingram CD, Taber DF, Oates JA, Brash AR (1982) Stereospecific removal of the DR hydrogen atom at the 10-carbon of arachidonic acid in the biosynthesis of leukotriene A4 by human leukocytes. J Biol Chem 257, 13515-9. 
MacMillan DK, Murphy RC (1995) Analysis of lipid hydroperoxides and long-chain conjugated keto acids by negative ion electrospray mass spectrometry. J Am Soc Mass Spectrom 6, 1190-201.

Malle E, Marsche G, Panzenboeck U, Sattler W (2006) Myeloperoxidase-mediated oxidation of highdensity lipoproteins: fingerprints of newly recognized potential proatherogenic lipoproteins. Arch Biochem Biophys 445, 245-55.

McDonald PP, McColl SR, Braquet P, Borgeat P (1994) Autocrine enhancement of leukotriene synthesis by endogenous leukotriene B4 and platelet-activating factor in human neutrophils. $\mathrm{Br} J$ Pharmacol 111, 852-60.

Morris JC (1966) Acid ionization constant of HOCl from 5 to 35 degrees. J Phys Chem 70, 3798-805.

Newcomer ME, Gilbert NC (2010) Location, location, location: compartmentalization of early events in leukotriene biosynthesis. J Biol Chem 285, 25109-14.

Pattison DI, Davies MJ (2001) Absolute rate constants for the reaction of hypochlorous acid with protein side chains and peptide bonds. Chem Res Toxicol 14, 1453-64.

Peers KE, Coxon DT (1983) Controlled synthesis of monohydroperoxides by alpha-tocopherol inhibited autoxidation of poly-unsaturated lipids. Chem Phys Lipids 32, 49-56.

Percival MD, Denis D, Riendeau D, Gresser MJ (1992) Investigation of the mechanism of nonturnover-dependent inactivation of purified human 5-lipoxygenase. Inactivation by $\mathrm{H} 2 \mathrm{O} 2$ and inhibition by metal ions. Eur J Biochem 210, 109-17.

Radmark O, Werz O, Steinhilber D, Samuelsson B (2015) 5-Lipoxygenase, a key enzyme for leukotriene biosynthesis in health and disease. Biochim Biophys Acta 1851, 331-9.

Riendeau D, Falgueyret JP, Nathaniel DJ, Rokach J, Ueda N, Yamamoto S (1989) Sensitivity of immunoaffinity-purified porcine 5-lipoxygenase to inhibitors and activating lipid hydroperoxides. Biochem Pharmacol 38, 2313-21.

Rouzer CA, Matsumoto T, Samuelsson B (1986) Single protein from human leukocytes possesses 5lipoxygenase and leukotriene A4 synthase activities. Proc Natl Acad Sci USA 83, 857-61.

Rouzer CA, Samuelsson B (1986) The importance of hydroperoxide activation for the detection and assay of mammalian 5-lipoxygenase. FEBS Lett 204, 293-6.

Salavej P, Spalteholz H, Arnhold J (2006) Modification of amino acid residues in human serum albumin by myeloperoxidase. Free Radic Biol Med 40, 516-25.

Schilstra MJ, Veldink GA, Verhagen J, Vliegenthart JF (1992) Effect of lipid hydroperoxide on lipoxygenase kinetics. Biochemistry 31, 7692-9.

Schwarz K, Walther M, Anton M, Gerth C, Feussner I, Kuhn H (2001) Structural basis for lipoxygenase specificity. Conversion of the human leukocyte 5-lipoxygenase to a 15-lipoxygenating enzyme species by site-directed mutagenesis. J Biol Chem 276, 773-9.

Smyrniotis CJ, Barbour SR, Xia Z, Hixon MS, Holman TR (2014) ATP allosterically activates the human 5-lipoxygenase molecular mechanism of arachidonic acid and 5(S)-hydroperoxy-6(E),8(Z),11(Z),14(Z)eicosatetraenoic acid. Biochemistry 53, 4407-19.

Walther M, Roffeis J, Jansen C, Anton M, Ivanov I, Kuhn H (2009) Structural basis for pH-dependent alterations of reaction specificity of vertebrate lipoxygenase isoforms. Biochim Biophys Acta 1791, 827-35.

Zschaler J, Arnhold J (2014) The hydroperoxide moiety of aliphatic lipid hydroperoxides is not affected by hypochlorous acid. Chem Phys Lipids 184, 42-51. 